\title{
Análise das principais afecções no trato digestivo inferior, através de colonoscopia, em clínica do Espírito Santo, entre 2013 e 2018
}

Analysis of the main affections in the lower digestive tract, through colonoscopy, in a clinic of Espírito Santo, between 2013 and 2018

Análisis de las principales afecciones del tracto digestivo inferior, mediante colonoscopia, en una clínica de Espírito Santo, entre 2013 y 2018

Amanda Rodrigues Lessa ${ }^{1 *}$, Rhainer Victor Simmer Schunk ${ }^{1}$, Guilherme Rodrigues Lessa ${ }^{1}$, Willian de Sá Lessa², Ana Rosa Murad Szpilman¹.

\section{RESUMO}

Objetivo: Estimar a prevalência das principais afecções que acometem o trato digestivo inferior, através de colonoscopia, em clínica do Espírito Santo, entre 2013 e 2018. Métodos: Trata-se de um estudo observacional descritivo e retrospectivo, onde foram avaliados 4.497 laudos digitais colonoscópios, realizados entre 14/02/2013 e 27/02/2018, enfatizando as variáveis: idade, gênero e afecção. Resultados: Dos 4.497 exames analisados, $1889(42,00 \%)$ eram normais, com $71,51 \%$ pacientes do sexo feminino, e $28,49 \%$ pacientes do sexo masculino, com idades entre 8 e 105 anos e média de 49,45 114,47 . Doença inflamatória intestinal $(7,20 \%)$, foi prevalente em mulheres, predominantemente entre 20 a 40 anos $(\mathrm{P}<0,05)$; Doença diverticular dos cólons (21,88\%), prevalência em mulheres e $>80$ anos $(P<0,05)$; Pólipos (14,40\%), prevalente em mulheres e $>50$ anos $(P<0,05)$; Hemorroidas $(18,74 \%)$ mulheres, faixa etária (FE) 45 a 65 anos $(p<0,05)$. Conclusão: Logo percebe-se que a população mais acometida é a feminina, por maior busca do sistema de saúde e as doenças apresentam uma prevalência distribuída consoante com o encontrado na literatura.

Palavras-chave: Epidemiologia, Colonoscopia, Trato digestivo, Prevalência, Prevenção de doenças.

ABSTRACT
Objective: To estimate the prevalence of the main conditions affecting the lower digestive tract, through
colonoscopy, in a clinic in Espírito Santo, between 2013 and 2018 . Methods: This is a descriptive and
retrospective observational study, where 4,497 digital colonoscope reports were evaluated, carried out
between $02 / 14 / 2013$ and $02 / 27 / 2018$, emphasizing the variables: age, gender and affection. Results: Of the
4,497 exams analyzed, $1889(42.00 \%)$ were normal, with $71.51 \%$ female patients, and $28.49 \%$ male patients,
aged between 8 and 105 years and a mean of $49.45 \pm 14.47$. Inflammatory bowel disease $(7.20 \%)$ was
prevalent in women, predominantly between 20 and 40 years $(P<0.05)$; Colonic diverticular disease $(21.88 \%)$,
prevalence in women and $>80$ years $(P<0.05) ;$ Polyps $(14.40 \%)$, prevalent in women aged $>50$ years $(P<0.05)$;
Hemorrhoids (18.74\%) women, age group (EF) 45 to 65 years $(p<0.05)$. Conclusion: Soon it is noticed that
the most affected population is the female, due to greater search of the health system and diseases have a
distributed prevalence according to what is found in the literature.

Key words: Epidemiology, Colonoscopy, Digestive tract, Prevalence, Disease prevention.

\section{RESUMEN}

Objetivo: Estimar la prevalencia de las principales afecciones que afectan el tracto digestivo inferior, mediante colonoscopia, en una clínica de Espírito Santo, entre 2013 y 2018. Métodos: Se trata de un estudio observacional descriptivo y retrospectivo, donde se evaluaron 4.497 informes de colonoscopios digitales. realizado entre 14/02/2013 y 27/02/2018, destacando las variables: edad, sexo y afecto. Resultados: De los 4.497 exámenes analizados, $1.889(42,00 \%)$ fueron normales, con un $71,51 \%$ de mujeres y un $28,49 \%$ de varones, con edades comprendidas entre 8 y 105 años y una media de $49,45 \pm 14,47$. La enfermedad inflamatoria intestinal $(7,20 \%)$ fue prevalente en mujeres, predominantemente entre 20 y 40 años $(P<0,05)$;

\footnotetext{
${ }^{1}$ Universidade Vila Velha (UVV), Vila Velha - ES. *E-mail: Amanda.r-|@hotmail.com
}

${ }^{2}$ Clínica MedLessa, Vila Velha - ES. 
Enfermedad diverticular colónica $(21,88 \%)$, prevalencia en mujeres y> 80 años $(P<0,05)$; Pólipos $(14,40 \%)$, prevalentes en mujeres $>50$ años $(P<0,05)$; Hemorroides $(18,74 \%)$ mujeres, grupo de edad (FE) de 45 a 65 años $(p<0,05)$. Conclusión: Pronto se advierte que la población más afectada es la femenina, debido a una mayor búsqueda del sistema de salud y las enfermedades tienen una prevalencia distribuida de acuerdo a lo encontrado en la literatura.

Palabras clave: Epidemiología, Colonoscopia, Tracto digestivo, Prevalencia, Prevención de enfermedades.

\section{INTRODUÇÃO}

Sabe-se que o trato digestivo inferior é sítio de patologias de diversas etiologias, por exemplo, as inflamatórias, como a Doença de Crohn e a Retocolite ulcerativa, as vasculares, as de distúrbios de motilidade, as infecciosas, as doenças funcionais e, além destas, as neoplasias, por isso, é necessário que se façam cada vez mais estudos sobre a prevalência e incidências dessas moléstias, afim de diminuir a morbimortalidade e os gastos públicos com essas questões (GOLDMAN L e SCHAFER Al, 2014).

A técnica que apresenta uma maior acurácia para diagnosticar lesões estruturais do cólon, especialmente neoplasias, é a colonoscopia. A colonoscopia apresenta diversas utilizações, sendo amplamente realizada em pacientes que apresentam sintomas no trato digestivo inferior, usada como ferramenta de triagens, diagnóstica, complementar a achados radiológicos duvidosos, como forma de propedêutica (após a retossigmoidoscopia) para hematoquezia, entre outras aplicações (LIEBERMAN D, 2012).

De acordo com a literatura, o sexo feminino é o mais acometido por doenças do trato digestivo inferior em relação ao sexo masculino e as faixas etárias mais prevalentes para o aparecimento de doença diverticular dos colons, pólipos colônicos, doença hemorroidária e doença inflamatória intestinal, que serão os 4 subgrupos focados no presente estudo, são, respectivamente, acima de 80 anos, 50 anos, 45 aos 65 anos e 20 aos 40 anos (PEMBERTON JH, et al, 2021; FINLAY AM, et al,. 2021; BLEDAY R, et al., 2020; MACRAE $A F$, et al., 2021).

As principais afecções em exames colonoscópios são, em ordem decrescente, doença diverticular dos cólons, pólipos de cólon, câncer colorretal e retocolite ulcerativa (PEMBERTON JH, et al., 2021; DOUBENI C, et al., 2020). Sobre a prevalência dessas enfermidades, de acordo com estudos, $50 \%$ das pessoas com idade entre 80 e 90 anos tem doença diverticular dos cólons (DOUBENI C, et al., 2020). A prevalência da doença hemorroidária, considerada elevada, é de difícil avaliação, sendo encontrados na literatura estudos epidemiológicos que apresentam valores discrepantes, desde 4,4\% a 86\%, conforme o tipo de populações estudadas (RAY-OFFOR E, AMADI S, 2019).

Em relação às moléstias poliposas, afirma-se que são altamente prevalentes, mas sua importância pautase na prevenção de câncer colorretal, uma vez que surge a partir da malignização de um pólipo adenomatoso, que correspondem a cerca de $70 \%$ dos pólipos e de acordo com o INCA, é sabido que, na contemporaneidade, o câncer de colorretal é o terceiro tipo mais frequente de neoplasia em homens e 0 segundo entre as mulheres, aumentando progressivamente com o passar dos anos (MINISTÉRIO DA SAÚDE, 2019).

Além do fato das condições serem prevalentes, há importantes complicações a serem consideradas como o desenvolvimento de câncer no caso dos pólipos ou tromboses, os estrangulamentos, as hemorragias e as ulcerações com infecção secundária e os abscessos no caso de hemorroida, entre outras complicações das doenças intestinais (MINISTÉRIO DA SAÚDE, 2019). Posto isso, objetivou-se estimar a prevalência das principais afecções que acometem o trato digestivo inferior, através de colonoscopia, em uma clínica do Espírito Santo, entre 2013 e 2018.

\section{MÉTODOS}

Estudo observacional, descritivo e retrospectivo. Foi analisada a prevalência das principais doenças no trato digestivo inferior, de uma amostra de 4.497 exames colonoscópios realizados em uma clínica no período de $14 / 02 / 2013$ a $27 / 02 / 2018$. 
Realizou-se levantamento de todos os laudos de colonoscopia no referido período e foram obtidas as seguintes variáveis: idade, gênero, afecção presente no documento. Foram considerados critérios de exclusão: dados sem resposta, resultados inconclusivos e colonoscopias parciais, retirando da amostra inicial de 4.640, 143 exames.

Calculou-se a prevalência, estratificada por gênero e idade, e para a análise estatística inferencial, foi utilizado o programa SPSS $\AA$ for Windows, versão 26.0, por meio do teste do qui-quadrado, para se averiguar a existência de associação, considerando $P<0,05$ como significativamente estatístico.

A pesquisa foi aprovada pelo Comitê de Ética em Pesquisa (CEP) da UVV sob o número 2.679.615 e foram respeitados os aspectos éticos da pesquisa com seres humanos de acordo com a Resolução 196/96 do Conselho Nacional de Saúde. Uma vez que o estudo foi observacional de dados digitalizados e não houve contato com pacientes, nem exposição de fotografias ou revelados dados de identificação, não foi aplicado o Termo de Consentimento Livre e Esclarecido (TCLE).

\section{RESULTADOS}

Foram analisados 4.497 exames colonoscópicos feitos em clínica particular do Espírito Santo no período de 14/02/2013 a 27/02/2018, sendo que 1705 (37,91\%) destes exames foram realizados por um único examinador. Dos 4497 exames analisados, foram encontrados $1889(42,00 \%)$ colonoscopias sem quaisquer alterações (normais), sendo 1351 (71,51\%) do sexo feminino e $538(28,48 \%)$ do sexo masculino. A média de idade foi de 49,45 $\pm 14,47$ anos com extremos de 8 e 105 anos.

Dos 2608 exames que apresentaram colonoscopias alteradas, 10,18\% eram Doença Inflamatória Intestinal, 30,95\% Doença Diverticular dos Cólons, 20,36\% Pólipos, 26,49\% Doença Hemorroidária e 12\% outros achados, classificados como "outras afecções".

Em um panorama geral, em relação a predominância do sexo nas principais doenças do estudo, temos doença hemorroidária, $533(63,22 \%)$ do sexo feminino e $310(36,77 \%)$ do sexo masculino; da doença diverticular dos cólons (DCC) 593 (60,20\%) eram mulheres e 392 (39,79\%) homens; já os pólipos intestinais correspondiam $350(54,01 \%)$ ao sexo feminino e 298 (45,98\%) ao masculino; por fim as doenças inflamatórias intestinais (DII) perfizeram um total de 194 (59,87\%) do sexo feminino e $130(40,12 \%)$ do masculino.

Em relação à doença diverticular dos cólons, pelo teste do qui-quadrado foi possível observar uma associação estatisticamente significativa da doença com a idade $(P<0,05)$, bem como para a idade-pólipos $(P<0,05)$ e idade-doença inflamatória intestinal $(p<0,05)$ (Tabela 1).

Tabela 1 - Distribuição de faixa etária com prevalência das principais doenças intestinais.

\begin{tabular}{lcc}
\hline Doença Hemorroidária & Número & Porcentagem \\
\hline$<45$ anos & 252 & 29.89 \\
$45-65$ anos & 465 & 55.15 \\
$>65$ anos & 126 & 14.94 \\
\hline Total & 843 & 100 \\
\hline Doença Diverticular dos Cólons & & 50.45 \\
\hline$<60$ anos & 497 & 45.48 \\
$60-80$ anos & 448 & 4.06 \\
$>80$ anos & 40 & 100 \\
\hline Total & 985 & \\
\hline Pólipos intestinais & & 33.33 \\
\hline$<50$ anos & 216 & 66.66 \\
$>50$ anos & 432 & 100 \\
\hline Total & 648 & \\
\hline Doença Inflamatória Intestinal & & 45.67 \\
\hline$<40$ anos & 148 & 54.32 \\
$>40$ anos & 176 & 100
\end{tabular}


O qui-quadrado não demonstrou associação do tipo de hemorroida (externa, interna ou mista) com o sexo ou com a faixa etária, porém a presença ou ausência da hemorroida mostrou-se associada à idade $(p<0,05)$. A Doença Inflamatória Intestinal (DII) totaliza 324 moléstias, sendo $194(60,00 \%)$ de forma isolada e 130 $(40,00 \%)$ associadas a outras doenças (Tabela 2$)$.

Tabela 2 - Doença inflamatória intestinal isolada ou associada a outras doenças.

\begin{tabular}{lcc}
\hline Doença inflamatória intestinal & Número & Porcentagem \\
\hline Proctite & 108 & 33.33 \\
Colite & 68 & 20.98 \\
Ileíte & 18 & 5.55 \\
\hline Associação com outras doenças & Número & Porcentagem \\
\hline Pólipo & 11 & 3.39 \\
Doença diverticular dos cólons & 43 & 13.27 \\
Hemorroida & 37 & 11.41 \\
Outros & 39 & 12.03 \\
\hline Total & 324 & $100 \%$ \\
\hline
\end{tabular}

Fonte: Lessa AR, et al., 2021.

Da amostra, foram encontrados $22(0,067 \%)$ achados macroscópicos sugestivos de retocolite ulcerativa, em que 12 (54,54\%) pertenciam ao sexo feminino e 10 (45,45\%) ao masculino, e $4(0,012 \%)$ de doença de Chron, sendo 1 (25\%) feminino e $3(75 \%)$ masculinos, porém como não era foco do trabalho, não foi investigado o resultado histopatológico dessas lesões. Os demais achados de lesões erosivas da mucosa são de aparência inespecífica. Das erosões sem aparência macroscópica específica podemos destacar a proctite, colite e ileíte.

Em geral são doenças de caráter benigno sem complicações. Dessas moléstias, em nosso estudo, foram analisados 324 laudos, destes, 194 (59,69\%) feminino e 130 (40,12\%) masculino. Não havendo, pelo teste do qui-quadrado, uma associação estatisticamente significante com o sexo $(p>0,05)$. Na faixa etária inferior a 20 anos, 11 (3,39\%) casos; na faixa entre 20 a 40 anos, 137 (42,28\%) pacientes; acima de 40 anos, 176 $(54,32 \%)$ casos.

Da amostra total, proctite isolada representou $108(33,23 \%)$ casos em que $68(62,96 \%)$ femininos e 40 $(37,03 \%)$ masculinos; ileíte $18(0,05 \%)$ casos com 14 (78\%) do sexo feminino e $4(22 \%)$ masculino; colite 68 $(0,20 \%)$ casos sendo $45(66,2 \%)$ do sexo feminino e $23(33,8 \%)$ do sexo masculino. Doença inflamatória intestinal associada a pólipo, doença diverticular dos cólons, hemorroida e outros somaram $130(40,12 \%)$ casos. A Doença diverticular dos cólons totalizou 985 (21,88\%) casos em que 593 (59,18\%) são mulheres e $392(40,81 \%)$ são homens, não demonstrando associação com o sexo pelo teste do qui-quadrado $(p>0,05)$.

Pólipos totalizaram 648 achados (Tabela 3). Dos pólipos encontrados de forma isolada, os segmentos mais acometidos foram respectivamente: reto 102 (38,63\%), cólon descendente 50 (18,93\%), cólon transverso $35(13,25 \%)$, sigmoide $32(12,12 \%)$, cólon ascendente $17(6,43 \%)$, ceco $14(5,30 \%)$, canal anal 8 (3,03\%) e ângulo hepático 6 (2,27\%). Diferentemente das moléstias anteriores, a associação pólipo-sexo se mostrou significativamente estatística pelo teste do qui-quadrado $(p<0,05)$.

Tabela 3 - Distribuição de pólipos em forma isolada ou associada a outras moléstias.

\begin{tabular}{lcc}
\hline Distribuição dos pólipos & Número & Porcentagem \\
\hline De forma isolada & 264 & 40.74 \\
Associado somente a outros pólipos & 56 & 8.64 \\
Associado à hemorróida & 68 & 10.49 \\
Associado à Doença Inflamatória Intestinal & 11 & 1.69 \\
Associado à Doença Diverticular dos Cólons & 171 & 26.38 \\
Associado à Doença Diverticular dos Cólons e Hemorroida & 28 & 4.32 \\
Associado a outras comorbidades & 50 & 7.71 \\
\hline Total & 648 & 100 \\
\hline
\end{tabular}

Fonte: Lessa AR, et al., 2021. 
Hemorroidas somam 843 exames analisados em que 533 (63,22\%) eram do sexo feminino e $310(36,77 \%)$ masculino. Do total, $463(54,92 \%)$ hemorroidas encontradas de forma isolada sendo que $316(68,25 \%)$ eram sexo feminino e 147 (31,74\%) masculino; e 380 (45,07\%) associadas com outras doenças, em que 220 $(57,89 \%)$ do sexo femininos e $160(42,10 \%)$ masculinos. O teste do qui-quadrado demonstrou associação hemorroida-gênero do paciente $(p<0,05)$.

A Tabela 4 apresenta outras afecções analisadas. Foram encontradas 91 lesões ulcero-vegetantes (LUV), sendo $48(52,74 \%)$ do sexo feminino e $43(47,25 \%)$ do sexo masculino. Vale ressaltar que a etiologia dessas lesões deve ser elucidada histologicamente.

Tabela 4 - Número de demais afecções analisadas.

\begin{tabular}{lcc}
\hline Afecção & Número & Porcentagem \\
\hline Angiodisplasia colônica & 53 & 13.87 \\
Cicatriz anal & 13 & 3.40 \\
Colectomia/Colostomia & 55 & 14.39 \\
Cólon espástico & 2 & 0.52 \\
Condiloma de canal anal & 1 & 0.26 \\
Dermatite perianal & 5 & 1.30 \\
Elevação de mucosa colônica & 26 & 6.80 \\
Endometriose & 1 & 0.26 \\
Endoparasitose & 2 & 0.52 \\
Estenose de cólon & 8 & 2.09 \\
Fissura anal & 27 & 7.06 \\
Fístula anal & 13 & 3.40 \\
Hiperplasia linfoide & 32 & 8.37 \\
Lesões Ulcerovegetantes & 91 & 23.82 \\
Lipoma de cólon & 14 & 3.66 \\
Megacólon & 2 & 0.52 \\
Melanose colônica & 8 & 2.09 \\
Micronodulações de cólon & 20 & 5.23 \\
Plicoma anal & 9 & 2.35 \\
\hline Total & 382 & 100 \\
\hline
\end{tabular}

Fonte: Lessa AR, et al., 2021.

Dos 143 exames excluídos, de acordo com os critérios definidos nos métodos, foram encontradas 78 $(54,54 \%)$ colonoscopias parciais por preparo irregular, das quais $49(62,82 \%)$ do sexo feminino e $29(37,17 \%)$ do sexo masculino, cerca de 20 (13,98\%) exames inconclusivos ou com dados faltosos e $45(31,46 \%)$ dólicocólon, dos quais $37(82,22 \%)$ do sexo feminino e $8(17,77 \%)$ do sexo masculino.

\section{DISCUSSÃO}

O estudo epidemiológico das doenças que acometem o trato digestivo inferior tem um significado clínico importante na diminuição da incidência e diagnóstico precoce dessas moléstias, isso porque sabendo onde a população carece de mais cuidados, é possível desenvolver programas visando à prevenção do desenvolvimento dessas (GOLDMAN L e SCHAFER Al, 2014; SIEGEL RL, et al., 2021).

Neste estudo, não foi possível coletar informações acerca dos motivos pelos quais o paciente buscou 0 serviço de saúde, bem como informações de IMC, hábitos alimentares e comorbidades pelo fato desses dados estarem arquivados em papel, juntamente com todos os dados endoscópicos dos anos anteriores. Mas, apesar dessas limitações, acreditamos ter alcançado um substrato importante para auxiliar na criação de projetos preventivos para a população.

Uma vez que as patologias pautadas no presente estudo já são bem conhecidas e explanadas na comunidade científica, bem como seus aspectos fisiopatológicos, etiopatogênicos e fatores de risco, foi encontrada uma limitação referente à temporalidade das últimas publicações, sendo elas mais antigas. As novas publicações baseiam-se em fatores epidemiológicos, apoiando-se ainda nas publicações lançadas há mais tempo, haja visto que as patologias permanecem as mesmas. 
Posto isso, e, salientando que nosso trabalho é de caráter epidemiológico, visando uma possível intervenção nas futuras prevalências dessas moléstias, expandimos o recorte temporal para apresentarmos os achados epidemiológicos do nosso estado, esperando contribuir com outros estudos desta natureza

\section{Doença Diverticular dos Cólons (DDC)}

A doença diverticular consiste em diverticulose e diverticulite, aumentando a prevalência com idade, numa proporção de $5 \%$ até 40 anos, 30\% com 60 anos e $65 \%$ na faixa dos 80 anos, podendo atingir até $80 \%$ da população idosa, sendo que nos grupos mais jovens a maior frequência se dá no sexo masculino. Também afirma que os sítios mais acometidos são respectivamente o sigmoide e/ou o cólon descendente (LIU PH, et al., 2017).

$\mathrm{Na}$ amostra do presente estudo, foi possível contemplar que, para a faixa etária inferior a 60 anos, teve uma prevalência de 291 (29,57\%) casos para o sexo feminino e 206 (20,93\%) para o sexo masculino. Para os encontrados entre 60 à 80 anos, $283(28,76 \%)$ para o sexo feminino e $165(16,76 \%)$ para o sexo masculino. Já os acima de 80 anos, 19 (01,92\%) do sexo feminino e $21(02,13 \%)$ do sexo masculino. Considerando as mulheres, somente $21,62 \%$ foram diagnosticadas e os homens $19,44 \%$.

Existem mais mulheres na amostra, compondo maior proporção dentre os resultados positivos e negativos para o exame, chamando atenção para o fato que as mulheres são as que mais buscam serviços de saúde porque homens associam a ideia de buscar o serviço como fragilidade, ferimento de ego e que o ambiente é mais propício para mulheres, crianças e idosos (LEVORATO CD, et al., 2014).

Pelo teste do qui-quadrado foi possível observar uma associação estatisticamente significativa da doença com a idade $(P<0,05)$, porém não houve uma significância em relação ao sexo $(p>0,05)$. Embora estudos demonstrassem não haver associação entre o sexo e o desfecho "desenvolver DDC" (STRATE LL e MORRIS AM, 2019), alguns autores encontraram correlação nos resultados de suas pesquisas (LEVORATO CD, et al., 2014).

Vale salientar que a prevalência aumenta com a idade, concordando com os resultados estatísticos do teste do qui-quadrado, onde, proporcionalmente e considerando toda a amostra, $14 \%$ dos pacientes tiveram o diagnóstico de doença diverticular dos cólons com menos de 60 anos, 47,1\% dos pacientes para a faixa etária entre 60 a 80 anos e $59,7 \%$ para a faixa etária de mais de 80 , revelando que dentro de cada faixa etária encontramos maior prevalência dentre os mais idosos (STRATE LL e MORRIS AM, 2019).

\section{Pólipos}

O pólipo intestinal é uma moléstia extremamente comum do intestino, secundária a um crescimento anormal da mucosa colônica e retal, afetando $15-20 \%$ da população e está correlacionado, em muitos casos, com o desenvolvimento do câncer colorretal (CCR) se não diagnosticado e tratado precocemente. (MACRAE AF, et al., 2021; FINLAY AM, et al., 2021).

Sabe-se que a incidência do CCR aumenta com a idade, sendo raramente encontrada em pacientes mais jovens, e acomete de igual modo ambos os sexos. No reto, o acometimento é mais comum em homens do que em mulheres. Também é conhecido o fato de pacientes com histórico familiar apresentarem um risco maior para o desenvolvimento deste câncer, sendo este aumento estimado entre 16-25\% para homens e 10$15 \%$ para mulheres. Todos esses constituem os principais fatores de risco (KASTENBERG D, et al., 2018; SIMON K, 2016).

Dos 648 exames analisados, $350(54,0 \%)$ eram do sexo feminino e $298(46,0 \%)$ do sexo masculino, o que está de acordo com a literatura, onde em um estudo realizado houve uma proporção de $50,8 \%$ do sexo feminino para $49,2 \%$ do sexo masculino (LEE L, et al., 2021). Quanto a idade, estudos realizados concluíram que a idade de maior risco para o surgimento destas alterações (mutações) se inicia após os 50 anos, sendo o ponto de corte definido para o início das colonoscopias de rastreio (MACRAE AF, et al., 2021; SIEGEL RL, et al., 2021).

Na presente pesquisa $432(66,7 \%)$ laudos revelaram pólipo em pacientes acima de 50 anos, enquanto 216 $(33,3 \%)$ abaixo de 50 anos, concordando com a literatura (MACRAE AF, et al., 2021), e com significância 
estatística pelo qui-quadrado $(P<0,05)$. Foi possível observar então que a incidência de pólipos aumenta com a idade, isso se deve a alterações cromossomais durante a vida, portanto quanto mais idoso, maiores as chances de tal alteração.

Uma vez que entendemos a epidemiologia, podemos partir para um rastreamento precoce mais agressivo, uma vez que a remoção desses pólipos interrompe a progressão para o câncer, elevando assim, a taxa de sobrevida desses pacientes em cinco anos, em aproximadamente $90 \%$ se tratado adequadamente (LEE L, et al., 2021; SIMON K, 2016).

\section{Doença Inflamatória Intestinal (DII)}

A retocolite ulcerativa e doença de Crohn são as principais doenças inflamatórias intestinais, caracterizadas por inflamação crônica no trato gastrointestinal (MALIK TA, 2015). A discussão em questão abordará a epidemiologia destas antes mencionadas, além de proctite, colite e ileíte, sendo essas três últimas de aparência macroscópica inespecífica. A proctite pode ser resultado da Doença de Chron, colite ulcerativa, radioterapia, secundária a uma infecção sexualmente transmissível (gonorreia, sífilis, infecção por Chlamydia trachomatis ou infecção por herpes simples), sobretudo em homossexuais do sexo masculino (PEPPERCORN MA, et al., 2021).

Já ileíte apresenta causas como processos autoinflamatórios, infecciosos (salmonelas, shigellas, enterococos e citomegalovírus) ou medicamentosos (anti-inflamatórios, anti-agregantes plaquetários). Apresenta vários graus de gravidade, mas é uma doença tratável (PEPPERCORN MA, et al., 2021). Outras causas de colite incluem infecções (Shigella dysenteriae e Escherichia coli, Clostridium difficile, entre outros), causas vasculares (colite isquêmica), e causas desconhecidas (colite linfocítica, colite microscópica e enterocolite necrosante) (WEDRO B, 2017).

Sobre os fatores de risco envolvidos na predisposição dessas enfermidades, a genética, fatores ambientais, vida sexual, infecções intestinais, higiene, agentes microbianos, dieta, cigarro, ocupação, poluição e estresse são os mais comumente citados (MALIK TA, 2015).

Apesar de em nossa análise mostrar uma maior concentração de pacientes na faixa acima dos 40 anos (176 casos, $54,3 \%$ ) se analisarmos de forma proporcional, $11,8 \%$ dos pacientes de toda a amostra foram diagnosticados na faixa etária abaixo dos 40 anos, concordando com o proposto na literatura em que a faixa prevalente compreende dos 20 aos 40 anos, enquanto $4,9 \%$ dos pacientes foram diagnosticados acima dos 50 anos. Considerando aspectos estatísticos, nossos achados também encontraram correlação significativa com a enfermidade e a idade do paciente $(P<0,05)$ (PEPPERCORN MA, et al., 2021)

\section{Doença hemorroidária}

A hemorroida é uma moléstia secundária à dilatação das veias anorretais, que ocorre por uma pressão persistentemente elevada nesses vasos, podendo acometer ambos os sexos e sendo mais prevalente entre os 45 a 65 anos (BRADLEY RD, et al., 2019).

Os fatores de risco para a presente enfermidade são dieta pobre em fibras, hiperplasia vascular dos corpos cavernosos do canal anal, prolapso anormal do plexo hemorroidário interno, força durante a evacuação, comunicações arteriovenosas muito calibrosas e disfunção do esfíncter anal interno, que contribuem para 0 aparecimento da hemorroida (JACOBS DO, 2018).

$\mathrm{Na}$ amostra do presente estudo foi possível inferir que a faixa etária inferior aos 45 anos teve uma prevalência de $252(29,9 \%)$ casos, sendo destes $162(64,28 \%)$ do sexo feminino e 90 (35,72\%) do sexo masculino. Consoante à literatura, a faixa etária que compreende dos 45 aos 65 anos foi mais proeminente apresentando prevalência de 465 (55,2\%) casos em que 294 (63,22\%) são do sexo feminino e 171 (36,77\%) são do sexo masculino. Acima dos 65 anos houve 126 (14,90\%) casos sendo destes 77 (61,11\%) do sexo feminino e $49(38,88 \%)$ do sexo masculino. Analisando todas as faixas etárias, o sexo feminino apresentou prevalência de 63,2\% (BRADLEY RD, et al., 2018),

A prevalência de hemorroida externa isolada foi de 144 (31,10\%), de hemorroida interna $178(38,44 \%)$ e de hemorroida mista $141(30,45 \%)$. O qui-quadrado não demonstrou associação do tipo de hemorroida 
(externa, interna ou mista) com o sexo ou com a fixa etária, porém a presença ou ausência da hemorroida mostrou-se associada ao sexo e à idade $(\mathrm{p}<0.05)$ pelo teste do qui-quadrado.

\section{CONCLUSÃO}

Consoante à análise dos dados epidemiológicos e da prevalência das moléstias encontrada nesse estudo, pode-se inferir que o sexo feminino foi no geral o público mais acometido pelas doenças (lesão ulcerovegetantes, pólipos, hemorroidas, doença diverticular dos cólons, doença inflamatória intestinal) e, para cada enfermidade separadamente, nosso estudo esteve de acordo com os dados encontrados na literatura no que tange à faixa etária.

\section{REFERÊNCIAS}

1. BLEDAY R, et al. Hemorrhoids: Clinical manifestations and diagnosis. UpToDate, 2020.

2. BRADLEY RD, et al. The American Society of Colon and Rectal Surgeons Clinical Practice Guidelines for the Management of Hemorrhoids. Diseases of the colon \& Rectum, 2018; 61(3)

3. BRASIL. Ministério da Saúde. Instituto Nacional de Câncer (INCA). Estatística para câncer de Intestino, Brasil, 2019. Disponível em: https://www.inca.gov.br/tipos-de-cancer/cancer-de-intestino. Acessado em: 26 de agosto de 2019.

4. DOUBENI C, et al., 2020. Tests for screening for colorectal câncer. UpToDate, 2020.

5. FINLAY AM, et al. Overview of colon polyps. UpToDate, 2021.

6. GOLDMAN L, SCHAFER AI Goldman Cecil Medicina: Tratado de Medicina Interna. 24.ed. Rio de Janeiro: Elsevier, 2014.

7. JACOBS DO. Hemorrhoids: what are the options in 2018?. Curr Opin Gastroenterol., 2018; 34(1): 46.

8. KASTENBERG D, et al. Bowel preparation quality scales for colonoscopy. World J Gastroenterol. 2018; 14: 24(26).

9. LEE L, et al. Overview of colonoscopy in adults. UpToDate, 2021.

10. LEVORATO CD, et al. Fatores associados à procura por serviços de saúde numa perspectiva relacional de gênero. Ciênc. saúde coletiva, 2014; 19(4): 1263-74.

11. LIU PH, et al. Adherence to a Healthy Lifestyle is Associated With a Lower Risk of Diverticulitis among Men. Am J Gastroenterol., 2017;112(12):1868.

12. MACRAE AF, et al. Clinical presentation, diagnosis, and staging of colorectal câncer. UpToDate, 2021.

13. MALIK TA. Inflammatory Bowel Disease: Historical Perspective, Epidemiology, and Risk Factors. Surg Clin North Am., 2015; 95(6):1105-22

14. PEMBERTON JH, et al. Clinical manifestations and diagnosis of acute diverticulitis in adults. UpToDate, 2021.

15. PEMBERTON JH, et al. Colonic diverticulosis and diverticular disease: Epidemiology, risk factors, and pathogenesis. UpToDate, 2021.

16. PEPPERCORN MA, et al., Definitions, epidemiology, and risk factors for inflammatory bowel disease. UpToDate, 2021.

17. RAY-OFFOR E, AMADI S. Hemorrhoidal disease: Predilection sites, pattern of presentation, and treatment. Ann Afr Med., 2019; 18(1): 12-16.

18. SIEGEL RL, et al. Cancer Statistics, 2021. CA Cancer J Clin., 2021; 71(1): 7.

19. SIMON K. Colorectal cancer development and advances in screening. Clin Interv Aging, 2016; 19(11): 967-76.

20. STRATE LL, MORRIS AM. Epidemiology, Pathophysiology, and Treatment of Diverticulitis. Gastroenterology, 2019; 156(5): 1282-1298.

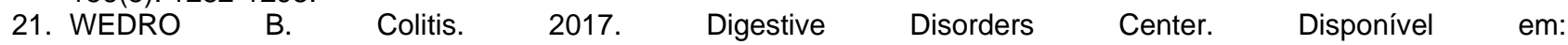
https://www.emedicinehealth.com/colitis/article_em.htm\#facts_about_and_definition_of_colitis. Acesso em 19 jul 2019. 